\title{
Dynamics of a second-order nonlinear difference system with exponents
}

\author{
D. S. Dilip ${ }^{1,2^{*}}$ and Smitha Mary Mathew ${ }^{1,2}$
}

\section{${ }^{*}$ Correspondence:}

dilip@stjohns.ac.in

${ }^{1}$ Department

of Mathematics, St. John's

College, Anchal, Kerala, India

Full list of author information

is available at the end of the

article

\begin{abstract}
In this paper, we study the persistence, boundedness, convergence, invariance and global asymptotic behavior of the positive solutions of the second-order difference system $\begin{aligned} x_{n+1} & =\alpha_{1}+a e^{-x_{n-1}}+b y_{n} e^{-y_{n-1}}, \\ y_{n+1} & =\alpha_{2}+c e^{-y_{n-1}}+d x_{n} e^{-x_{n-1}} \quad n=0,1,2, \ldots\end{aligned} \quad$ where $\alpha_{1}, \alpha_{2}, a, b, c, d$ are positive real numbers and the initial conditions $x_{-1}, x_{0}, y_{-1}, y_{0}$ are arbitrary nonnegative numbers.
\end{abstract}

Keywords: Local behavior, Global behavior, Invariance, Persistence, Boundedness

AMS Subject Classification: $39 \mathrm{~A} 22$

\section{Introduction}

The theory of discrete dynamical system has many applications in applied sciences. Mathematical modeling of a physical, biological or ecological problem mostly leads to a nonlinear difference system. (See [1-10].)

In [4], Papachinopoulos et al. proposed a system of equation with exponents as

$$
f_{n+1}=a+b f_{n-1} e^{-g_{n}}, g_{n+1}=c+d g_{n-1} e^{-f_{n}}, \quad n=0,1,2, \ldots,
$$

where $a, b, c, d$ and the initial conditions $f_{-1}, f_{0}, g_{-1}, g_{0}$ are positive real values. They studied the existence, boundedness and asymptotic behavior of the positive solutions of (1).

In [5], G.Papaschinopoulos and C.J.Schinas together modified the system as

$$
\begin{aligned}
& f_{n+1}=a+b g_{n-1} e^{-f_{n}}, g_{n+1}=c+\mathrm{d} f_{n-1} e^{-g_{n}}, \\
& f_{n+1}=a+b g_{n-1} e^{-g_{n}}, g_{n+1}=c+\mathrm{d} f_{n-1} e^{-f_{n}},
\end{aligned}
$$

and put forward conditions for the positive solutions to be asymptotic.

In [11], authors multiplied $f_{n}$ and $g_{n}$ with $a$ and $c$, respectively, in (2) and formed a new system of difference equations

$$
f_{n+1}=a f_{n}+b g_{n-1} e^{-f_{n}}, g_{n+1}=c g_{n}+\mathrm{d} f_{n-1} e^{-g_{n}}, n=0,1, \ldots
$$
priate credit to the original author(s) and the source, provide a link to the Creative Commons licence, and indicate if changes were made. The images or other third party material in this article are included in the article's Creative Commons licence, unless indicated otherwise in a credit line to the material. If material is not included in the article's Creative Commons licence and your intended use is not permitted by statutory regulation or exceeds the permitted use, you will need to obtain permission directly from the copyright holder. To view a copy of this licence, visit http://creativecommons.org/licenses/by/4.0/. 
and described the existence of a unique positive equilibrium, the boundedness, persistence and global attractivity of the positive solutions.

Parallelly in [12], the authors worked on the asymptotic behavior of the positive solutions of a similar difference system

$$
f_{n+1}=a g_{n}+b f_{n-1} e^{-g_{n}}, g_{n+1}=c f_{n}+\mathrm{d} g_{n-1} e^{-f_{n}}, n=0,1, \ldots
$$

N.Psarros and G.Papaschinopoulos in [13] proposed a new first-order model

$$
f_{n+1}=a g_{n}+b f_{n} e^{-f_{n}-g_{n}}, g_{n+1}=c f_{n}+\mathrm{d} g_{n} e^{-f_{n}-g_{n}},
$$

and studied the asymptotic behavior of the positive solutions of the system.

Motivated by the above research articles, we propose a new second order difference system

$$
\begin{aligned}
& x_{n+1}=\alpha_{1}+a e^{-x_{n-1}}+b y_{n} e^{-y_{n-1}}, \\
& y_{n+1}=\alpha_{2}+c e^{-y_{n-1}}+d x_{n} e^{-x_{n-1}} \quad n=0,1,2, \ldots
\end{aligned}
$$

where $\alpha_{1}, \alpha_{2}, a, b, c, d$ are positive real numbers and the initial conditions $x_{-1}, x_{0}, y_{-1}, y_{0}$ are arbitrary nonnegative numbers, and investigate the persistence, boundedness, convergence, invariance, and global asymptotic behavior of the positive solutions of the system.

\section{Methods}

We use Theorem 1.16 of [14] to prove the lemma which we use to derive a condition for the existence, uniqueness of equilibrium solutions and the convergence of positive solutions to the equilibrium solution. We also use Remark 1.3.1 of [15] to obtain conditions for global asymptotic stability of the unique equilibrium point.

\section{Results and discussion}

The following theorem proposes conditions for persistence and boundedness for the positive solution $\left(x_{n}, y_{n}\right)$ of (3).

Theorem 1 Every positive solution $\left(x_{n}, y_{n}\right)$ of (3) is bounded and persists whenever $b d e^{-\alpha_{1}-\alpha_{2}}<1$.

Proof

$x_{n} \geq \alpha_{1}, y_{n} \geq \alpha_{2}, n=3,4, \ldots$.

Hence, $\left(x_{n}, y_{n}\right)$ of system (3) persists.

Also, (3) becomes

$$
\begin{aligned}
x_{n+1} & \leq \alpha_{1}+a e^{-\alpha_{1}}+b e^{-\alpha_{2}}\left[\alpha_{2}+\mathrm{d} x_{n-1} e^{-x_{n-2}}+c e^{-y_{n-2}}\right] . \\
& \leq A+b d x_{n-1} e^{-\alpha_{1}-\alpha_{2}}
\end{aligned}
$$

where $A=\alpha_{1}+a e^{-\alpha_{1}}+b \alpha_{2} e^{-\alpha_{2}}+b c e^{-\alpha_{2}-\alpha_{2}}$. 
Similarly,

$$
y_{n+1} \leq C+b d y_{n-1} e^{-\alpha_{1}-\alpha_{2}}
$$

where $C=\alpha_{2}+c e^{-\alpha_{2}}+d \alpha_{1} e^{-\alpha_{1}}+a d e^{-\alpha_{1}-\alpha_{1}}$.

Now, consider the difference equations

$$
\begin{aligned}
& z_{n+1}=A+B z_{n-1} . \\
& v_{n+1}=C+D v_{n-1},
\end{aligned}
$$

where $B=D=b d e^{-\alpha_{1}-\alpha_{2}}<1$. Therefore, an arbitrary solution $\left(z_{n}, v_{n}\right)$ of (6) can be written as

$$
\begin{aligned}
& z_{n}=r_{1} B^{n / 2}+r_{2}(-1)^{n} B^{n / 2}+\frac{A}{1-B}, \quad n=0,1,2, \ldots \\
& v_{n}=s_{1} B^{n / 2}+s_{2}(-1)^{n} B^{n / 2}+\frac{C}{1-B}, \quad n=0,1,2, \ldots
\end{aligned}
$$

where $r_{1}, r_{2}$ rely on the initial conditions $z_{-1}, z_{0}$ and $s_{1}, s_{2}$ rely on the initial conditions $v_{-1}, v_{0}$. Hence, $\left(z_{n}, v_{n}\right)$ is bounded.

Let us examine the solution $\left(z_{n}, v_{n}\right)$ such that $z_{-1}=x_{-1}, z_{0}=x_{0}, v_{-1}=y_{-1}, v_{0}=y_{0}$.

Hence by induction, $x_{n} \leq z_{n}$ and $y_{n} \leq v_{n}, n=0,1,2, \ldots$

Therefore, we get $\left(x_{n}, y_{n}\right)$ is bounded.

The following two theorems confirm the existence of invariant boxes of (3).

Theorem 2 Let $b d e^{-\alpha_{1}-\alpha_{2}}<1$. Let $\left(x_{n}, y_{n}\right)$ denote a positive solution of (3). Then $\left[\alpha_{1}, \frac{\alpha_{1}+a e^{-\alpha_{1}}+b \alpha_{2} e^{-\alpha_{2}}+b c e^{-\alpha_{2}-\alpha_{2}}}{\left(1-b d e^{-\alpha_{1}-\alpha_{2}}\right)}\right] \times\left[\alpha_{2}, \frac{\alpha_{2}+c e^{-\alpha_{2}}+d \alpha_{1} e^{-\alpha_{1}}+a d e^{-\alpha_{1}-\alpha_{1}}}{\left(1-b d e^{-\alpha_{1}-\alpha_{2}}\right)}\right]$ is an invariant set for (3).

Proof

Let

$$
I_{1}=\left[\alpha_{1}, \frac{\alpha_{1}+a e^{-\alpha_{1}}+b \alpha_{2} e^{-\alpha_{2}}+b c e^{-\alpha_{2}-\alpha_{2}}}{\left(1-b d e^{-\alpha_{1}-\alpha_{2}}\right)}\right]
$$

$I_{2}=\left[\alpha_{2}, \frac{\alpha_{2}+c e^{-\alpha_{2}}+d \alpha_{1} e^{-\alpha_{1}}+a d e^{-\alpha_{1}-\alpha_{1}}}{\left(1-b d e^{-\alpha_{1}-\alpha_{2}}\right)}\right]$.

Let $x_{-1}, x_{0} \in I_{1}$ and $y_{-1}, y_{0} \in I_{2}$.

Then

$$
\begin{aligned}
x_{1} & \leq \alpha_{1}+a e^{-\alpha_{1}}+b e^{-\alpha_{2}} y_{0} \\
& \leq \alpha_{1}+a e^{-\alpha_{1}}+b e^{-\alpha_{2}}\left[\frac{\alpha_{2}+c e^{-\alpha_{2}}+d \alpha_{1} e^{-\alpha_{1}}+a d e^{-\alpha_{1}-\alpha_{1}}}{1-b d e^{-\alpha_{1}-\alpha_{2}}}\right] .
\end{aligned}
$$


Hence, we get $x_{1} \leq \frac{\alpha_{1}+a e^{-\alpha_{1}}+b \alpha_{2} e^{-\alpha_{2}}+b c e^{-\alpha_{2}-\alpha_{2}}}{1-b d e^{-\alpha_{1}-\alpha_{2}}}$. i.e., $x_{1} \in I_{1}$. Similarly, we get $y_{1} \in I_{2}$.

Hence, the proof follows by applying the method of induction.

Theorem 3 Let bde $e^{-\alpha_{1}-\alpha_{2}}<1$. Consider the intervals

$$
I_{3}=\left[\alpha_{1}, \frac{\alpha_{1}+a e^{-\alpha_{1}}+b \alpha_{2} e^{-\alpha_{2}}+b c e^{-\alpha_{2}-\alpha_{2}}+\epsilon}{1-b d e^{-\alpha_{1}-\alpha_{2}}}\right]
$$

and

$$
I_{4}=\left[\alpha_{2}, \frac{\alpha_{2}+c e^{-\alpha_{2}}+d \alpha_{1} e^{-\alpha_{1}}+a d e^{-\alpha_{1}-\alpha_{1}}+\epsilon}{1-b d e^{-\alpha_{1}-\alpha_{2}}}\right]
$$

where $\epsilon$ is an arbitrary positive number. If $\left(x_{n}, y_{n}\right)$ is any arbitrary solution of (3), then there exists an $N \in \mathbb{N}$ such that $x_{n} \in I_{3}$ and $y_{n} \in I_{4}, n \geq N$.

Proof

Let $\left(x_{n}, y_{n}\right)$ denote an arbitrary solution of (3).

Then by Theorem $1, \lim \sup _{n \rightarrow \infty} x_{n}=M<\infty$ and $\lim \sup _{n \rightarrow \infty} y_{n}=L<\infty$.

Hence from Theorem 1, $x_{n+1} \leq A+b d x_{n-1} e^{-\alpha_{1}-\alpha_{2}}$ and $y_{n+1} \leq C+b d y_{n-1} e^{-\alpha_{1}-\alpha_{2}}$

Hence $M \leq \frac{A}{1-b d e^{-\alpha_{1}-\alpha_{2}}}$, and $L \leq \frac{C}{1-b d e^{-\alpha_{1}-\alpha_{2}}}$.

Hence, there exists an $N \in \mathbb{N}$ such that the theorem holds.

Now we prove a lemma which is an alteration of Theorem 1.16 of [14].

Lemma 4 Let $[a, b]$ and $[c, d]$ denote intervals of real numbers. Let $f:[a, b] \times[c, d] \times[c, d] \rightarrow[a, b]$ and $g:[a, b] \times[a, b] \times[c, d] \rightarrow[c, d]$ be continuous functions. Consider the difference system

$$
\begin{aligned}
& x_{n+1}=f\left(x_{n-1}, y_{n}, y_{n-1}\right), \\
& y_{n+1}=g\left(x_{n}, x_{n-1}, y_{n-1}\right), \quad n=0,1,2, \ldots
\end{aligned}
$$

such that the initial values $x_{-1}, x_{0} \in[a, b]$ and $y_{-1}, y_{0} \in[c, d]$. (or $x_{n_{0}}, x_{n_{0}+1} \in[a, b]$, $\left.y_{n_{0}}, y_{n_{0}+1} \in[c, d], n_{0} \in \mathbb{N}\right)$. Suppose the following are true.

1. If $f(x, y, z)$ is nonincreasing in $x, f(x, y, z)$ is nondecreasing in $y$ and $f(x, y, z)$ is nonincreasing in $z$.

2. If $g(x, y, z)$ is nondecreasing in $x, g(x, y, z)$ is nonincreasing in $y$ and $g(x, y, z)$ is nonincreasing in $z$. 
3. If $\left(m_{1}, M_{1}, m_{2}, M_{2}\right) \in[a, b]^{2} \times[c, d]^{2}$ satisfies the systems $m_{1}=f\left(M_{1}, m_{2}, M_{2}\right)$, $M_{1}=f\left(m_{1}, M_{2}, m_{2}\right)$ and $m_{2}=g\left(m_{1}, M_{1}, M_{2}\right), M_{2}=g\left(M_{1}, m_{1}, m_{2}\right)$ then $M_{1}=m_{1}$ and $M_{2}=m_{2}$,

then there exists a unique equilibrium solution $(\bar{x}, \bar{y})$ of (9) with $\bar{x} \in[a, b], \bar{y} \in[c, d]$. Also every solution of (9) converges to $(\bar{x}, \bar{y})$.

Proof

Set $m_{1}^{-1}=a, m_{1}^{0}=a, m_{2}^{-1}=c, m_{2}^{0}=c$.

$$
M_{1}^{-1}=b, M_{1}^{0}=b, M_{2}^{-1}=d, M_{2}^{0}=d \text {. }
$$

For each $i \geq 0$, let $m_{1}^{i+1}=f\left(M_{1}^{i-1}, m_{2}^{i}, M_{2}^{i-1}\right), M_{1}^{i+1}=f\left(m_{1}^{i-1}, M_{2}^{i}, m_{2}^{i-1}\right)$ and

$$
m_{2}^{i+1}=g\left(M_{1}^{i}, m_{1}^{i-1}, m_{2}^{i-1}\right), M_{2}^{i+1}=g\left(m_{1}^{i}, M_{1}^{i-1}, M_{2}^{i-1}\right) .
$$

Hence $m_{1}^{1}=f\left(M_{1}^{-1}, m_{2}^{0}, M_{2}^{-1}\right) \leq f\left(m_{1}^{-1}, M_{2}^{0}, m_{2}^{-1}\right)=M_{1}^{1}$, and

$$
m_{2}^{1}=g\left(m_{1}^{0}, M_{1}^{-1}, M_{2}^{-1}\right) \leq g\left(M_{1}^{0}, m_{1}^{-1}, m_{2}^{-1}\right)=M_{2}^{1} .
$$

Therefore,

$$
\begin{aligned}
& M_{1}^{-1} \geq M_{1}^{0} \geq M_{1}^{1} \geq m_{1}^{1} \geq m_{1}^{0} \geq m_{1}^{-1} \quad \text { and } \\
& M_{2}^{-1} \geq M_{2}^{0} \geq M_{2}^{1} \geq m_{2}^{1} \geq m_{2}^{0} \geq m_{2}^{-1} .
\end{aligned}
$$

Also $m_{1}^{0}=a \leq x_{n} \leq b=M_{1}^{0}, n \geq 0$ and $m_{2}^{0}=c \leq y_{n} \leq d=M_{2}^{0}, n \geq 0$.

For all $n \geq 0$, we have

$$
\begin{aligned}
& m_{1}^{1}=f\left(M_{1}^{-1}, m_{2}^{0}, M_{2}^{-1}\right) \leq f\left(x_{n-1}, y_{n}, y_{n-1}\right) \leq f\left(m_{1}^{-1}, M_{2}^{0}, m_{1}^{-1}\right)=M_{1}^{1} . \\
& m_{2}^{1}=g\left(m_{1}^{0}, M_{1}^{-1}, M_{2}^{-1}\right) \leq g\left(x_{n}, x_{n-1}, y_{n-1}\right) \leq g\left(M_{1}^{0}, m_{1}^{-1}, M_{2}^{0}\right)=M_{2}^{1} .
\end{aligned}
$$

Hence $m_{1}^{1} \leq x_{n} \leq M_{1}^{1}, n \geq 1$ and $m_{2}^{1} \leq y_{n} \leq M_{2}^{1}, n \geq 1$.

We then obtain by induction that for $i \geq 0$, the following are true.

1. $a=m_{1}^{-1} \leq m_{1}^{0} \leq m_{1}^{1} \ldots \leq m_{1}^{i-1} \leq m_{1}^{i} \leq M_{1}^{i} \ldots \leq M_{1}^{1} \leq M_{1}^{0} \leq M_{1}^{-1}=b$.

2. $c=m_{2}^{-1} \leq m_{2}^{0} \leq m_{2}^{1} \ldots \leq m_{2}^{i-1} \leq m_{2}^{i} \leq M_{2}^{i} \ldots \leq M_{2}^{1} \leq M_{2}^{0} \leq M_{2}^{-1}=d$.

3. $m_{1}^{i} \leq x_{n} \leq M_{1}^{i}, n \geq 1$ and $m_{2}^{i} \leq y_{n} \leq M_{2}^{i}, n \geq 1$.

Set $m_{1}=\lim _{i \rightarrow \infty} m_{1}^{i}, m_{2}=\lim _{i \rightarrow \infty} m_{2}^{i}$ and $M_{1}=\lim _{i \rightarrow \infty} M_{1}^{i}, M_{2}=\lim _{i \rightarrow \infty} M_{2}^{i}$.

Since $f$ and $g$ are continuous, we get $m_{1}=f\left(M_{1}, m_{2}, M_{2}\right), M_{1}=f\left(m_{1}, M_{2}, m_{2}\right)$ and $m_{2}=g\left(m_{1}, M_{1}, M_{2}\right), M_{2}=g\left(M_{1}, m_{1}, m_{2}\right)$.

Hence $M_{1}=m_{1}=\bar{x}$ and $M_{2}=m_{2}=\bar{y}$, from which we get the proof.

The following theorem proposes conditions for the convergence of the equilibrium solution of (3). 


\section{Theorem 5 Suppose}

$$
b d e^{-\alpha_{1}-\alpha_{2}}<1, c e^{-\alpha_{2}}<1, a e^{-\alpha_{1}}<1
$$

and

$$
\begin{gathered}
\frac{b d e^{-\alpha_{1}-\alpha_{2}}}{\left[1-b d e^{-\alpha_{1}-\alpha_{2}}\right]^{2}} \frac{\left[1-b d e^{-\alpha_{1}-\alpha_{2}}+\alpha_{2}+c e^{-\alpha_{2}}+\mathrm{d} \alpha_{1} e^{-\alpha_{1}}+a d e^{-\alpha_{1}-\alpha_{1}}\right]}{\left[1-a e^{-\alpha_{1}}\right]} \\
\times \frac{\left[1-b d e^{-\alpha_{1}-\alpha_{2}}+\alpha_{1}+a e^{-\alpha_{1}}+b \alpha_{2} e^{-\alpha_{2}}+b c e^{-\alpha_{2}-\alpha_{2}}\right]}{\left[1-c e^{-\alpha_{2}}\right]}<1 .
\end{gathered}
$$

Then (3) has a unique positive equilibrium $E(\bar{x}, \bar{y})$. Also, every solution of (3) converges to $E(\bar{x}, \bar{y})$.

\section{Proof}

Let $f: \mathbb{R}^{+} \times \mathbb{R}^{+} \times \mathbb{R}^{+} \rightarrow \mathbb{R}^{+}, g: \mathbb{R}^{+} \times \mathbb{R}^{+} \times \mathbb{R}^{+} \rightarrow \mathbb{R}^{+}$be continuous functions such that $f(x, y, z)=\alpha_{1}+a e^{-x}+b y e^{-z}, g(x, y, z)=\alpha_{2}+c e^{-z}+d x e^{-y}$.

Let $M_{1}, m_{1}, M_{2}, m_{2}$ be positive real numbers satisfying

$$
m_{1}=\alpha_{1}+a e^{-M_{1}}+b m_{2} e^{-M_{2}}, M_{1}=\alpha_{1}+a e^{-m_{1}}+b M_{2} e^{-m_{2}}
$$

and

$$
m_{2}=\alpha_{2}+c e^{-M_{2}}+\mathrm{d} m_{1} e^{-M_{1}}, M_{2}=\alpha_{2}+c e^{-m_{2}}+\mathrm{d} M_{1} e^{-m_{1}} .
$$

Therefore, $M_{1}-m_{1}=a\left[e^{-m_{1}}-e^{-M_{1}}\right]+b\left[M_{2} e^{-m_{2}}-m_{2} e^{-M_{2}}\right]$.

$$
M_{1}-m_{1}=a\left[e^{-m_{1}}-e^{-M_{1}}\right]+b e^{-m_{2}-M_{2}}\left[M_{2} e^{M_{2}}-m_{2} e^{m_{2}}\right] .
$$

Also, there exists a $\zeta, m_{2} \leq \zeta \leq M_{2}$ satisfying

$$
M_{2} e^{M_{2}}-m_{2} e^{m_{2}}=(1+\zeta) e^{\zeta}\left(M_{2}-m_{2}\right) .
$$

From (13) and (14), we get

$$
M_{1}-m_{1}=a\left[e^{-m_{1}}-e^{-M_{1}}\right]+b e^{-m_{2}-M_{2}+\zeta}(1+\zeta)\left[M_{2}-m_{2}\right] .
$$

Now, $a\left[e^{-m_{1}}-e^{-M_{1}}\right]=a e^{-m_{1}-M_{1}}\left[e^{M_{1}}-e^{m_{1}}\right]$.

Also there exists a $\lambda, m_{1} \leq \lambda \leq M_{1}$ satisfying

$$
a\left[e^{-m_{1}}-e^{-M_{1}}\right]=a e^{-m_{1}-M_{1}+\lambda}\left[M_{1}-m_{1}\right] .
$$

Since $M_{1}, m_{1} \geq \alpha_{1}$ and $\lambda \leq M_{1}$,

$$
a\left[e^{-m_{1}}-e^{-M_{1}}\right] \leq a e^{-\alpha_{1}}\left[M_{1}-m_{1}\right] .
$$

Thus, from (15) and (17) we get, 


$$
M_{1}-m_{1} \leq a e^{-\alpha_{1}}\left[M_{1}-m_{1}\right]+b e^{-m_{2}-M_{2}+\zeta}(1+\zeta)\left[M_{2}-m_{2}\right]
$$

Since $M_{2}, m_{2} \geq \alpha_{2}$ and $\zeta \leq M_{2},(18)$ becomes

$$
M_{1}-m_{1} \leq a e^{-\alpha_{1}}\left[M_{1}-m_{1}\right]+b e^{-\alpha_{2}}(1+\zeta)\left[M_{2}-m_{2}\right]
$$

, i.e.,

$$
\left[1-a e^{-\alpha_{1}}\right]\left[M_{1}-m_{1}\right] \leq b e^{-\alpha_{2}}(1+\zeta)\left[M_{2}-m_{2}\right] .
$$

Also, (12) can be written as

$$
\begin{aligned}
& M_{2}=\alpha_{2}+c e^{-m_{2}}+\mathrm{d}\left[\alpha_{1}+a e^{-m_{1}}+b M_{2} e^{-m_{2}}\right] e^{-m_{1}} . \\
& M_{2} \leq \frac{\alpha_{2}+c e^{-\alpha_{2}}+\mathrm{d} \alpha_{1} e^{-\alpha_{1}}+a d e^{-\alpha_{1}-\alpha_{1}}}{1-b d e^{-\alpha_{1}-\alpha_{2}}} .
\end{aligned}
$$

Since $\zeta \leq M_{2}$ we get,

$$
\zeta \leq \frac{\alpha_{2}+c e^{-\alpha_{2}}+\mathrm{d} \alpha_{1} e^{-\alpha_{1}}+a d e^{-\alpha_{1}-\alpha_{1}}}{1-b d e^{-\alpha_{1}-\alpha_{2}}} .
$$

Therefore, (20) becomes

$$
\begin{aligned}
& {\left[1-a e^{-\alpha_{1}}\right]\left[M_{1}-m_{1}\right]} \\
& \quad \leq b e^{-\alpha_{2}}\left[\frac{\left.1-b d e^{-\alpha_{1}-\alpha_{2}}+\alpha_{2}+c e^{-\alpha_{2}}+\mathrm{d} \alpha_{1} e^{-\alpha_{1}}+a d e^{-\alpha_{1}-\alpha_{1}}\right]}{1-b d e^{-\alpha_{1}-\alpha_{2}}}\right]\left[M_{2}-m_{2}\right] .
\end{aligned}
$$

Similarly, we get

$$
\begin{aligned}
& {\left[1-c e^{-\alpha_{2}}\right]\left[M_{2}-m_{2}\right]} \\
& \quad \leq \mathrm{d} e^{-\alpha_{1}}\left[\frac{\left.1-b d e^{-\alpha_{1}-\alpha_{2}}+\alpha_{1}+a e^{-\alpha_{1}}+b \alpha_{2} e^{-\alpha_{2}}+b c e^{-\alpha_{2}-\alpha_{2}}\right]}{1-b d e^{-\alpha_{1}-\alpha_{2}}}\right]\left[M_{1}-m_{1}\right] .
\end{aligned}
$$

From (24) and (25), we get

$$
\begin{aligned}
{\left[M_{1}\right.} & \left.-m_{1}\right] \\
\leq & \frac{b d e^{-\alpha_{1}-\alpha_{2}}}{\left[1-\left(b d e^{\left.-\alpha_{1}-\alpha_{2}\right)}\right]^{2}\right.} \frac{\left[1-b d e^{-\alpha_{1}-\alpha_{2}}+\alpha_{2}+c e^{-\alpha_{2}}+\mathrm{d} \alpha_{1} e^{-\alpha_{1}}+a d e^{-\alpha_{1}-\alpha_{1}}\right]}{\left[1-a e^{-\alpha_{1}}\right]} \\
& \times \frac{\left[1-b d e^{-\alpha_{1}-\alpha_{2}}+\alpha_{1}+a e^{-\alpha_{1}}+b \alpha_{2} e^{-\alpha_{2}}+b c e^{-\alpha_{2}-\alpha_{2}}\right]}{\left[1-c e^{-\alpha_{2}}\right]}\left[M_{1}-m_{1}\right] .
\end{aligned}
$$

Therefore from (11) and (26), we get $M_{1}=m_{1}$ and $M_{2}=m_{2}$.

Therefore by applying Lemma 4, the result is obtained.

In the next theorem, we derive conditions for the global asymptotic stability of the equilibrium solution of (3).

Theorem 6 Assume (10) and (11) holds. 
1. Let $(a+a c+c)<1$. If $(1+\bar{x})(1+\bar{y})<\frac{1-(a+a c+c)}{b d}$, then the unique equilibrium $E(\bar{x}, \bar{y})$ is globally asymptotically stable.

2. If $(a+c+a c+b d)+b d\left[\frac{A}{1-B}+\frac{C}{1-B}+\frac{A C}{(1-B)^{2}}\right]<1$, where $A, B$ and $C$ are defined as in (4) and (5), then the unique equilibrium $E(\bar{x}, \bar{y})$ is globally asymptotically stable.

Proof

First we show that $E(\bar{x}, \bar{y})$ is locally asymptotically stable in both the cases. The Jacobian $J F(\bar{x}, \bar{y})$ about the equilibrium point $E(\bar{x}, \bar{y})$ is given by

$$
\left[\begin{array}{cccc}
0 & -a e^{-\bar{x}} & b e^{-\bar{y}} & -b \bar{y} e^{-\bar{y}} \\
1 & 0 & 0 & 0 \\
\mathrm{~d} e^{-\bar{x}} & -d \bar{x} e^{-\bar{x}} & 0 & -c e^{-\bar{y}} \\
0 & 0 & 1 & 0
\end{array}\right] .
$$

Hence the characteristic equation of the Jacobian $J F(\bar{x}, \bar{y})$ about the equilibrium point $E(\bar{x}, \bar{y})$ is given by

$$
\begin{aligned}
& -\lambda^{4}+\lambda^{2}\left(-c e^{-\bar{y}}+b d e^{-\bar{x}} e^{-\bar{y}}-a e^{-\bar{x}}\right) \\
& \quad+\lambda\left(-b d \bar{y} e^{-\bar{x}} e^{-\bar{y}}-b d \bar{x} e^{-\bar{x}} e^{-\bar{y}}\right)+b d \bar{x} \bar{y} e^{-\bar{x}} e^{-\bar{y}}-a c e^{-\bar{x}} e^{-\bar{y}}=0 .
\end{aligned}
$$

Then

$$
\begin{aligned}
& \left|-c e^{-\bar{y}}\right|+\left|b d e^{-\bar{x}} e^{-\bar{y}}\right|+\left|a e^{-\bar{x}}\right| \\
& \quad+\left|b d \bar{y} e^{-\bar{x}} e^{-\bar{y}}\right|+\left|b d \bar{x} e^{-\bar{x}} e^{-\bar{y}}\right|+\left|b d \bar{x} \bar{y} e^{-\bar{x}} e^{-\bar{y}}\right|+\left|a c e^{-\bar{x}} e^{-\bar{y}}\right|<1
\end{aligned}
$$

is satisfied whenever

$$
|c|+|b d|+|a|+|b d \bar{y}|+|b d \bar{x}|+|b d \bar{x} \bar{y}|+|a c|<1 .
$$

1. From (27), we get

$$
(1+\bar{x})(1+\bar{y})<\frac{1-(a+a c+c)}{b d} .
$$

Hence, by (28) and Remark 1.3.1 of [15], we get the result.

2. Since $E(\bar{x}, \bar{y})$ is the equilibrium point of (3), we get

$$
\bar{x} \leq \alpha_{1}+a e^{-\alpha_{1}}+b e^{-\alpha_{2}}\left[\alpha_{2}+d \bar{x} e^{-\alpha_{1}}+c e^{-\alpha_{2}}\right] .
$$

, i.e.,

$$
\bar{x} \leq \frac{A}{\left(1-b d e^{-\alpha_{1}-\alpha_{2}}\right)} .
$$

Similarly 


$$
\bar{y} \leq \frac{C}{\left(1-b d e^{-\alpha_{1}-\alpha_{2}}\right)}
$$

Substituting (29), (30) in (27), we get

$$
(a+c+a c+b d)+b d\left[\frac{A}{1-B}+\frac{C}{1-B}+\frac{A C}{(1-B)^{2}}\right]<1 .
$$

Hence by Remark 1.3.1 of [15], we get the result.

Therefore by using Theorem 5, we obtain the conditions for global asymptotic stability.

\section{Conclusions}

In this paper, we analyzed the persistence, boundedness, convergence, invariance and global asymptotic behavior of the positive solutions of a second-order difference system. Here we expressed all the conditions in terms of the parameters occurring in the system. We also obtained two conditions for the occurrence of global stability where in the first one the condition was given in terms of the equilibrium point and in the second one the condition was given in terms of parameters of the system.

Acknowledgements

The authors would like to thank the referees for their valuable suggestions.

\section{Authors' contributions}

DSD wrote the title, abstract, introduction and references. SMM wrote the main results. Both authors read and approved the final manuscript.

\section{Funding}

This research work is not funded.

\section{Availability of data and materials}

Not applicable.

\section{Declarations}

\section{Competing interests}

The authors declare that they have no competing interests.

\section{Author details}

${ }^{1}$ Department of Mathematics, St. John's College, Anchal, Kerala, India. ${ }^{2}$ Department of Mathematics, Mar Ivanios College, Research Centre, University of Kerala, Thiruvananthapuram, India.

Received: 23 September 2020 Accepted: 5 April 2021

Published online: 20 April 2021

\section{References}

1. Qureshi, M.N., Qadeer Khan, A., Din, Q.: Asymptotic behavior of a Nicholson-Bailey model. Adv. Differ. Equ. 2014, 62 (2014). https://doi.org/10.1186/1687-1847-2014-62

2. Clark, C.A., Kulenovic, M.R.S., Selgrade, J.F.: On a system of rational difference equations. J. Differ. Equ. Appl. 11(7), 565-580 (2005). https://doi.org/10.1080/10236190412331334464

3. Moranjkic, S., Nurkanovic, Z.: Basins of attraction of certain rational anti-competitive system of difference equations in the plane. Adv. Differ. Equ. 2012, 153 (2012). https://doi.org/10.1186/1687-1847-2012-153

4. Papaschinopoulos, G., Radin, M.A., Schinas, C.J.: On the system of two difference equations of exponential form. Math. Comput. Mod. 54, 2969-2977 (2011). https://doi.org/10.1016/j.mcm.2011.07.019

5. Papaschinopoulos, G., Schinas, C.J.: On the dynamics of two exponential type systems of difference equations. Comput. Math. Appl. 64, 2326-2334 (2012). https://doi.org/10.1016/j.camwa.2012.04.002

6. Din, Q.: Complexity and chaos control in a discrete-time prey-predator model. Commun. Nonliear Sci. Numer. Simul. 49, 113-134 (2017). https://doi.org/10.1016/j.cnsns.2017.01.025

7. Nurkanovic, M., Nurkanovic, Z.: Basins of attraction of an anti-competitive discrete rational system. Sarajevo J. Math. 8(21), 259-272 (2012). https://doi.org/10.5644/SMJ.08.2.07

8. Tilman, D., Wedin, D.: Oscillations and chaos in the dynamics of a perennial grass. Lett. Nat. 353, $653-655$ (1991) 
9. Papaschinopoulos, G., Schinas, C.J., Ellina, G.: On the dynamics of the solutions of a biological model. J. Differ. Equ. Appl. 20(5-6), 694-705 (2014). https://doi.org/10.1080/10236198.2013.806493

10. Feng, H., Ma, H., Ding, W.: Global asymptotic behavior of positive solutions for exponential form difference equations with three parameters. J. Appl. Anal. Comput. 6(3), 600-606 (2016). https://doi.org/10.11948/2016041

11. Papaschinopoulos, G., Ellina, G., Papadopoulos, K.B.: Asymptotic behavior of the positive solutions of an exponential type system of difference equations. Appl. Math. Comput. 245, 181-190 (2014). https://doi.org/10.1016/j.amc.2014. 07.074

12. Papaschinopoulos, G., Fotiades, N., Schinas, C.J.: On a system of difference equations including negative exponential terms. J. Differ. Equ. Appl. 20(5-6), 717-732 (2014). https://doi.org/10.1080/10236198.2013.814647

13. Psarros, N., Papaschinopoulos, G.: Long-term behavior of positive solutions of an exponentially self-regulating system of difference equations. Int. J. Biomath. 10(3), 1750045 (2017). https://doi.org/10.1142/S1793524517500450

14. Grove, E.A., Ladas, G.: Periodicities in Nonlinear Difference Equations. Chapman \& Hall, London (2005)

15. Kocic, V.L., Ladas, G.: Global Behavior of Nonlinear Difference Equations of Higher Order with Applications. Kluwer, Dordrecht (1993)

\section{Publisher's Note}

Springer Nature remains neutral with regard to jurisdictional claims in published maps and institutional affiliations.

\section{Submit your manuscript to a SpringerOpen ${ }^{\circ}$ journal and benefit from:}

- Convenient online submission

- Rigorous peer review

- Open access: articles freely available online

- High visibility within the field

Retaining the copyright to your article

Submit your next manuscript at $\boldsymbol{\Delta}$ springeropen.com 